\title{
Aleksandrov reflection and geometric evolution of hypersurfaces
}

\author{
Bennett Chow AND Robert Gulliver
}

\begin{abstract}
Consider a compact embedded hypersurface $\Gamma_{t}$ in $\mathbb{R}^{n+1}$ which moves with speed determined at each point by a function $F\left(\kappa_{1}, \ldots, \kappa_{n}, t\right)$ of its principal curvatures, for $0 \leq t<T$. We assume the problem is degenerate parabolic, that is, that $F(\cdot, t)$ is nondecreasing in each of the principal curvatures $\kappa_{1}, \ldots, \kappa_{n}$. We shall show that for $t>0$ the hypersurface $\Gamma_{t}$ satisfies local a priori Lipschitz bounds outside of a convex set determined by $\Gamma_{0}$ and lying inside its convex hull. Our method is the parabolic analogue of Aleksandrov's method of moving planes [A1], [A2], [A3], [A4], [AVo].

The flow of a smooth hypersurface may be generalized to the evolution of a closed set $\Gamma_{t}$ described as the level set of a continuous function $u_{t}$ which satisfies in the viscosity sense a degenerate parabolic PDE defined by $F$ for $0 \leq t<\infty$, [ES], [CGG]. It has recently been noted that this levelset flow, even when starting from a smooth hypersurface $\Gamma_{0}$, may develop a nonempty interior after the evolving hypersurface collides with itself or develops singularities [BP], [AIC], [AVe], [K]. We shall prove that the same local Lipschitz bounds as in the hypersurface case hold for the inner and outer boundaries of $\Gamma_{t}$.
\end{abstract}

As an application, we give some new results about $1 / H$ flow for nonstar-shaped hypersurfaces, which was recently investigated by Huisken and Ilmanen [HI]. We prove existence and asymptotic roundness, in the Lipschitz sense, for "extended" viscosity solutions in $\mathbb{R}^{n+1}$. In contrast, the evolving hypersurfaces given in [HI], which were used to prove a version of the Penrose conjecture, are solutions of a non-local variational problem, valid in general asymptotically flat Riemannian manifolds. 


\section{Main results.}

Let $\Gamma_{t}$ be a embedded connected $C^{2}$ hypersurface in $\mathbb{R}^{n+1}$ evolving in the direction of its unit inward normal vector $N$ with speed

$$
V=F\left(\kappa_{1}, \ldots, \kappa_{n}, t\right)
$$

a function of the principal curvatures $\kappa_{1} \leq \cdots \leq \kappa_{n}$ and time $t \in[0, T)$ where $0<T \leq \infty$. Here we assume that $F_{t}=F(\cdot, t)$ is uniformly Lipschitz continuous for each $t \geq 0$ and $F$ is non-decreasing in each $\kappa_{i}$, which ensures the evolution equation is degenerate parabolic (weakly parabolic). Our sign convention is such that $\kappa_{i}$ is positive on the standard sphere. We will not require that $F_{t}$ be symmetric.

Choose a unit vector $\nu \in S^{n}$ and a number $\lambda \in \mathbb{R}$. Define the hyperplane

$$
P=P^{\lambda}(\nu)=\left\{x \in \mathbb{R}^{n+1}:\langle x, \nu\rangle=\lambda\right\}
$$

and the half-spaces

$$
\begin{aligned}
& H_{+}=H_{+}^{\lambda}(\nu)=\{x:\langle x, \nu\rangle>\lambda\} \\
& H_{-}=H_{-}^{\lambda}(\nu)=\{x:\langle x, \nu\rangle<\lambda\} .
\end{aligned}
$$

We have the orthogonal reflection in $P^{\lambda}(\nu)$

$$
\sigma^{\lambda}(\nu)(x)=x-2(\langle x, \nu\rangle-\lambda) \nu .
$$

For simplicity, when the choice of $\lambda \in \mathbb{R}$ and $\nu \in S^{n}$ are clear, we denote $x^{*}=\sigma^{\lambda}(\nu)(x)$.

Any embedded connected $C^{2}$ hypersurface $\Gamma$ is the boundary of a connected bounded open set $\Omega, \Gamma=\partial \Omega$.

Definition 1. We say that $\lambda$ is admissible for $\Gamma$ with respect to $\nu$ if

$$
\sigma^{\lambda}(\nu)\left(\Gamma \cap H_{-}^{\lambda}(\nu)\right) \subset \bar{\Omega} .
$$

We have the following Aleksandrov reflection-type result.

Theorem 2. Let $\Gamma_{t}$ be a family of embedded $C^{2}$ hypersurfaces evolving by equation (1.1).

1. If $\lambda$ is admissible for $\Gamma_{0}$ with respect to $\nu$, then $\lambda$ is admissible for $\Gamma_{t}$ with respect to $\nu$ for all $t \in[0, T)$. 
2. If $\Gamma_{0}$ is compact and $(-\infty, \lambda)$ is admissible for $\Gamma_{0}$ with respect to all $\nu$ in the neighborhood of $\nu_{0}$, then $\Gamma_{t} \cap H_{-}^{\lambda}\left(\nu_{0}\right)$ is a $C^{2}$ graph in the direction $\nu_{0}$ with locally bounded gradient; this local bound is independent of $t$ and $F$.

For an example where the first part of Theorem 2 is valid but not the second part, see the example of section 4.2 below.

Let $\lambda_{\max }(\nu)$ be the supremum of all $\lambda$ such that $(-\infty, \lambda]$ is admissible for $\Gamma_{0}$ with respect to $\nu$. For convenience, we shall write $H_{ \pm}^{\max }(\nu)$ for the half-space $H_{ \pm}^{\lambda_{\max }(\nu)}(\nu)$. According to Theorem 2, Part (2), it will also be important to consider the upper semicontinuous envelope $\bar{\lambda}$ of $\lambda_{\max }: S^{n} \rightarrow$ $\mathbb{R}$.

Corollary 3. Let $K$ be the intersection of the closures of the halfspaces $H_{+}^{\bar{\lambda}(\nu)}(\nu)$, for $\nu \in S^{n}$. Then the part of the hypersurface $\Gamma_{t}$ lying outside $K$ satisfies local gradient bounds, which depend only on $\Gamma_{0}$.

Theorem 4. Let $\Lambda=\Lambda\left(\Gamma_{0}\right)=\sup _{\nu \in S^{n}}\left[-\lambda_{\max }(\nu)\right]$. Then for all $t \in[0, T)$, the part of the hypersurface $\Gamma_{t}$ lying outside the ball $\bar{B}_{\Lambda}(0)$ is a radial graph $r=\psi_{t}(\theta), \theta \in S^{n}$, with gradient estimate

$$
\left|D \psi_{t}(\theta)\right| \leq \frac{r \Lambda}{\sqrt{r^{2}-\Lambda^{2}}} .
$$

For example, if $\Gamma_{0}$ is close in the Lipschitz norm to a round sphere, then the constant $\Lambda$ of Theorem 4 is a small positive number, and for all $t>0$, the part of $\Gamma_{t}$ lying outside of $\bar{B}_{\Lambda}(0)$ will be Lipschitz close to a round sphere. In fact, $\log \left(\psi_{t} / \Lambda\right)$ satisfies Lipschitz estimates governed by its lower bound.

As another example, if $\Gamma_{t}$ leaves every compact set, it must become round in the sense that after rescaling to get $\left|\Omega_{t}\right|=\left|B_{1}\right|, \Gamma_{t}$ converges to the unit sphere in the Lipschitz norm. Note that in the literature, such convergence to a round sphere has been proven in the $C^{2}$ sense for certain strictly parabolic problems by first showing the curvatures all converge to 1 . Our methods, in contrast, work for degenerate parabolic problems and do not require (nor imply) the pointwise convergence of curvatures.

The results above extend to generalized solutions (viscosity solutions). In particular, let $\Gamma_{0}$ be an embedded compact (but not necessarily connected) $C^{0}$ hypersurface bounding a bounded open (not necessarily con- 
nected) set $\Omega_{0}$. Let $K>0$. Define $u_{0}: \mathbb{R}^{n+1} \rightarrow \mathbb{R}$ by

$$
u_{0}(x)=\left\{\begin{array}{ll}
\operatorname{dist}\left(x, \Gamma_{0}\right) \wedge K & \text { if } x \in \mathbb{R}^{n+1} \backslash \Omega_{0} \\
-\operatorname{dist}\left(x, \Gamma_{0}\right) & \text { if } x \in \Omega_{0}
\end{array},\right.
$$

which is the signed distance function to $\Gamma_{0}$ (cut off by the constant $K$.) Then $\Gamma_{0}=\left\{x: u_{0}(x)=0\right\}$. Consider the level set flow (see [ES] and [CGG]). That is, for $(x, t) \in \mathbb{R}^{n+1} \times[0, \infty)$ let $u_{t}(x)$ be the unique continuous solution of the degenerate parabolic equation

$$
\frac{\partial u_{t}}{\partial t}=-\left|D u_{t}\right| F\left(\kappa_{1}, \ldots, \kappa_{n}, t\right)
$$

where $\kappa_{1} \leq \cdots \leq \kappa_{n}$ are the eigenvalues of $D\left(\left|D u_{t}\right|^{-1} D u_{t}\right)$, with the given initial condition $u_{0}(x)$. The evolving zero-set (which is not necessarily a hypersurface) is given by

$$
\Gamma_{t}=\left\{x: u_{t}(x)=0\right\}
$$

$\Gamma_{t}$ is called the generalized solution to the evolution problem (1.1), and is unique. Recall that for $t>0, \Gamma_{t}$ is uniquely determined by $\Gamma_{0}$ independent of the choice of $u_{0}$. Also, since $\Gamma_{0}$ is compact, $\Gamma_{t}$ is a compact set for each $t \in[0, \infty)$. Define further

$$
\begin{aligned}
& \Omega_{t}=\left\{x: u_{t}(x)<0\right\} \\
& E_{t}=\left\{x: u_{t}(x)>0\right\} .
\end{aligned}
$$

We then have $\mathbb{R}^{n+1}=\Gamma_{t} \cup \Omega_{t} \cup E_{t}$ as a disjoint union. In fact (contrary to a conjecture stated for the case $F=\kappa_{1}+\cdots+\kappa_{n}$ by E. DeGiorgi, L.C. Evans and J. Spruck) a smooth hypersurface $\Gamma_{0}$ may evolve into a set $\Gamma_{t}$ with nonempty interior after a finite time. See $[\mathrm{BP}],[\mathrm{K}],[\mathrm{AVe}]$ and section 4 below.

Definition 5. Let $\Gamma, \Omega, E$ be disjoint sets such that $\mathbb{R}^{n+1}=\Gamma \cup \Omega \cup E$. Given $\nu \in S^{n}$ and $\lambda \in \mathbb{R}$, we say that $\lambda$ is admissible for the triple $(\Gamma, \Omega, E)$ with respect to $\nu$ if both

$$
\begin{aligned}
& \sigma^{\lambda}(\nu)\left(\Omega \cap H_{-}^{\lambda}(\nu)\right) \subset \Omega \cap H_{+}^{\lambda}(\nu) \\
& \sigma^{\lambda}(\nu)\left(E \cap H_{-}^{\lambda}(\nu)\right) \supset E \cap H_{+}^{\lambda}(\nu) .
\end{aligned}
$$


Since $\mathbb{R}^{n+1}=\Gamma \cup \Omega \cup E$ is a disjoint union, these containments are respectively equivalent to

$$
\begin{aligned}
& \sigma^{\lambda}(\nu)\left((E \cup \Gamma) \cap H_{-}^{\lambda}(\nu)\right) \supset(E \cup \Gamma) \cap H_{+}^{\lambda}(\nu) \\
& \sigma^{\lambda}(\nu)\left((\Omega \cup \Gamma) \cap H_{-}^{\lambda}(\nu)\right) \subset(\Omega \cup \Gamma) \cap H_{+}^{\lambda}(\nu) .
\end{aligned}
$$

When $\Gamma$ is an embedded compact connected $C^{2}$ hypersurface, $\Omega$ is the bounded open set with boundary $\Gamma$ and $E$ is their complement, this definition agrees with our previous definition of admissibility.

Theorem 6. Let $\Gamma_{t}$ be the zero-set of a solution $u_{t}$ to a degenerate parabolic geometric level-set flow.

1. If $\lambda$ is admissible for the triple $\left(\Gamma_{0}, \Omega_{0}, E_{0}\right)$ with respect to $\nu$, then $\lambda$ is admissible for the triple $\left(\Gamma_{t}, \Omega_{t}, E_{t}\right)$ with respect to $\nu$ for all $t \geq 0$.

2. If $\Gamma_{0}$ is compact and $\left(-\infty, \lambda_{0}\right)$ is admissible for the triple $\left(\Gamma_{0}, \Omega_{0}, E_{0}\right)$ with respect to all $\nu$ in a neighborhood of $\nu_{0}$, then $\Gamma_{t} \cap H_{-}^{\lambda_{0}}\left(\nu_{0}\right)$ is the closed set between two locally Lipschitz graphs in the direction $\nu_{0}$, with Lipschitz bounds independent of $F$ and of $t$.

Remark 1. It will be apparent to the reader that in the context of level-set solutions, the conclusions of Corollary 3 and of Theorem 4 hold for the inner boundary and for the outer boundary of the level-set solution $\Gamma_{t}$. Similarly, the following corollary is stated for level-set solutions, but applies to $\Gamma_{t}$ itself in the smooth case.

Corollary 7. Let $\Lambda:=\sup _{\nu \in S^{n}}\left[-\lambda_{\max }(\nu)\right]$ be determined by $\Gamma_{0}$. For $t \geq 0$, let $\partial_{0}\left(\Gamma_{t}\right)$ be a connected component of either the inner or the outer boundary of the level-set solution $\Gamma_{t}$. There is a constant $C_{0}=4.603$ such that if $\partial_{0}\left(\Gamma_{t}\right)$ contains a point $x_{0}$ with $\left|x_{0}\right|>C_{0} \Lambda$, then $\partial_{0}\left(\Gamma_{t}\right)$ is a radial graph in an annulus of width $\left(C_{0}-1\right) \Lambda$. Moreover, any other connected components of the inner or outer boundary (respectively) must lie inside $\overline{B_{\Lambda}(0)}$.

Remark 2. It will be seen from the proof that $C_{0}=\sqrt{\sigma^{2}+1}$, where $\sigma-$ $\arctan \sigma=\pi$, and that $\pi<C_{0}-1<3 \pi / 2$.

This paper is closely connected with the authors' 1996 paper [CG1]. In the special case that $\Gamma_{t}$ is a strictly convex hypersurface, it may be represented by its support function $v_{t}: S^{n} \rightarrow \mathbb{R}$. Then $v=v_{t}$ satisfies the 
evolution PDE

$$
\frac{\partial v}{\partial t}=G(\nabla \nabla v+v g, t)
$$

where, writing $G(\cdot, t)$ in terms of the eigenvalues of its matrix argument, $G\left(\lambda_{1}, \ldots, \lambda_{n}, t\right)=F\left(\left(\lambda_{1}\right)^{-1}, \ldots,\left(\lambda_{n}\right)^{-1}, t\right)$ and where $g$ is the standard Riemannian metric on $S^{n}$. For this special case, results such as Corollary 3 of the present paper follow from Theorem 3.1, part (iv) of [CG1].

\section{Proofs.}

We first define admissibility for functions on $\mathbb{R}^{n+1}$.

Definition 8. Let $u: \mathbb{R}^{n+1} \rightarrow \mathbb{R}$ be a continuous function. Given $\nu \in S^{n}$ and $\lambda \in \mathbb{R}$, we say that $\lambda$ is admissible for $u$ with respect to $\nu$ if

$$
u(x) \geq u\left(x^{*}\right) \quad \text { for all } x \in H_{-}^{\lambda}(\nu),
$$

where $x^{*}=\sigma^{\lambda}(\nu)(x)=x-2(\langle x, \nu\rangle-\lambda) \nu$.

Lemma 9. Let $\Gamma$ be the zero-set of a continuous function $u$. If $\lambda$ is admissible for $u$ with respect to $\nu$, then $\lambda$ is admissible for the triple $(\Gamma, \Omega, E)$ with respect to $\nu$, where $\Omega=\{x: u(x)<0\}$ and $E=\{x: u(x)>0\}$. If $u$ is the signed distance function, then the converse is true.

Proof. Write $H_{ \pm}=H_{ \pm}^{\lambda}(\nu)$. If $\lambda$ is admissible for $u$ with respect to $\nu$, then for all $x \in \Omega \cap H_{-}$we have $u\left(x^{*}\right) \leq u(x)<0$, which implies $x^{*} \in \Omega \cap H_{+}$. Similarly, if $x \in E \cap H_{+}$, then $u\left(x^{*}\right) \geq u(x)>0$, which implies $x^{*} \in E \cap H_{-}$. That is, $\lambda$ is admissible for the triple $(\Gamma, \Omega, E)$ with respect to $\nu$.

Conversely, suppose $\lambda$ is admissible for the triple $(\Gamma, \Omega, E)$ with respect to $\nu$. We want to show that the signed distance function $u(x) \geq u\left(x^{*}\right)$ for all $x \in H_{-}$.

First, suppose $x \in(E \cup \Gamma) \cap H_{-}$. If the closest point $y \in \Gamma$ to $x$ is in $H_{+}$, then $\left|x^{*}-y\right| \leq|x-y|$, so that $u\left(x^{*}\right) \leq u(x)$. Otherwise, $y$ is in $H_{-}$, in which case $y^{*} \in(\Omega \cup \Gamma) \cap H_{+}$. At the same time, $\sigma^{\lambda}(\nu)\left[(\Omega \cup \Gamma) \cap H_{-}\right] \subset$ $(\Omega \cup \Gamma) \cap H_{+}$, hence

$$
\begin{aligned}
u\left(x^{*}\right) & =\operatorname{dist}\left(x^{*},(\Omega \cup \Gamma)\right) \leq \operatorname{dist}\left(x^{*}, y^{*}\right) \\
& =\operatorname{dist}\left(x,(\Omega \cup \Gamma) \cap H_{-}\right)=u(x) .
\end{aligned}
$$


Second, suppose $x \in(\Omega \cup \Gamma) \cap H_{-}$. Now $\sigma^{\lambda}(\nu)\left((E \cup \Gamma) \cap H_{-}\right) \supset(E \cup$ Г) $\cap H_{+}$implies

$$
\begin{aligned}
\operatorname{dist}\left(x,(E \cup \Gamma) \cap H_{+}\right) & \geq \operatorname{dist}\left(x^{*},(E \cup \Gamma) \cap H_{+}\right) \\
& \geq \operatorname{dist}\left(x^{*}, \sigma^{\lambda}(\nu)\left[(E \cup \Gamma) \cap H_{-}\right]\right) \\
& =\operatorname{dist}\left(x,(E \cup \Gamma) \cap H_{-}\right),
\end{aligned}
$$

and hence

$$
\begin{aligned}
u(x) & =-\operatorname{dist}\left(x,(E \cup \Gamma) \cap H_{-}\right) \\
& \geq-\operatorname{dist}\left(x^{*},(E \cup \Gamma) \cap H_{+}\right)=u\left(x^{*}\right) .
\end{aligned}
$$

In particular, the lemma implies that if $u$ is the signed distance function of a $C^{2}$ hypersurface $\Gamma$, then $\lambda$ is admissible for $u$ with respect to $\nu$ if and only if $\lambda$ is admissible for $\Gamma$ with respect to $\nu$.

Hence Theorem 2 follows from Theorem 6 and we only give the

Proof of Theorem 6. Let $u_{0}$ be the signed distance function to $\Gamma_{0}$. Since $\lambda$ is admissible for the triple $\left(\Gamma_{0}, \Omega_{0}, E_{0}\right)$ with respect to $\nu$, the lemma implies $\lambda$ is admissible for $u_{0}$ with respect to $\nu$. That is, $u_{0}(x) \geq u_{0}\left(x^{*}\right)$ for all $x \in H_{-}^{\lambda}(\nu)$. For $x \in P^{\lambda}(\nu)$, we have $x=x^{*}$. Thus, by the maximum principle for viscosity solutions, the solution $u_{t}$ to the geometric level set flow satisfies $u_{t}(x) \geq u_{t}\left(x^{*}\right)$ for all $x \in H_{-}^{\lambda}(\nu)$ and $t \geq 0$. That is, $\lambda$ is admissible for $u_{t}$ with respect to $\nu$ for all $t \geq 0$. Applying the lemma again implies $\lambda$ is admissible for the triple $\left(\Gamma_{t}, \Omega_{t}, E_{t}\right)$ with respect to $\nu$ for all $t \geq 0$.

Before considering part (2) of Theorem 6, we shall prove the

Proposition 10. If $\left(-\infty, \lambda_{0}\right)$ is admissible for the function $u$ with respect to $\nu$, then $u$ is nonincreasing in the $\nu$ direction on $H_{-}^{\lambda_{0}}(\nu)$.

Proof. Let $y \in P^{\lambda_{0}}(\nu)$, and consider any two points $x=y+s \nu$ and $x^{\prime}=$ $y+s^{\prime} \nu$ with $s<s^{\prime} \leq 0$. Their bisecting hyperplane is $P^{\lambda}(\nu)$, where $\lambda=$ $\lambda_{0}+\frac{1}{2}\left(s+s^{\prime}\right)<\lambda_{0}$, and we have $x \in H_{-}^{\lambda}(\nu)$, while

$$
\begin{aligned}
x^{*} & :=x-2(\langle x, \nu\rangle-\lambda) \nu=y+s \nu-2\left(\lambda_{0}+s-\lambda\right) \nu \\
& =y+\left(2 \lambda-2 \lambda_{0}-s\right) \nu=y+s^{\prime} \nu=x^{\prime} .
\end{aligned}
$$

So the first part of Theorem 6 shows $u_{t}(x) \geq u_{t}\left(x^{*}\right)$, that is, $u_{t}$ is nonincreasing in the $\nu$-direction when restricted to the half-space $H_{-}^{\lambda_{0}}(\nu)$. 
Proof of Theorem 6, Part (2). Let $\varepsilon$ be the radius in $S^{n}$ of a ball so that $\left(-\infty, \lambda_{0}\right)$ is admissible for $\left(\Gamma_{0}, \Omega_{0}, E_{0}\right)$ with respect to each $\nu$ in the ball. As we have shown above, $\left(-\infty, \lambda_{0}\right)$ is also admissible for $u_{t}$ with respect to each $\nu$ in the ball. It now follows from Proposition 10 that $u_{t}$ is nonincreasing in the $\nu$ direction on $H_{-}^{\lambda_{0}}(\nu)$ for all $\nu$ with $\operatorname{dist}\left(\nu, \nu_{0}\right)<\varepsilon$.

Consider two points $\hat{x}, \tilde{x} \in H_{-}^{\lambda_{0}}\left(\nu_{0}\right)$ which are in the outer boundary $\partial_{\text {out }}\left(\Gamma_{t}\right):=\Gamma_{t} \cap \overline{E_{t}}$. Then there are $\hat{x}_{k} \rightarrow \hat{x}$ with $u_{t}\left(\hat{x}_{k}\right)>0$ for all $k$. Since $H_{-}^{\lambda_{0}}\left(\nu_{0}\right)$ is an open halfspace, we have $\hat{x}, \tilde{x} \in H_{-}^{\lambda_{0}}(\nu)$ for all $\nu \in S^{n}$ with $\operatorname{dist}\left(\nu, \nu_{0}\right)<\hat{\varepsilon}$ for some $\hat{\varepsilon} \in(0, \varepsilon]$ which depends on $\hat{x}$ and $\tilde{x}$. Write $\hat{x}=\hat{y}+\hat{s} \nu_{0}$ and $\tilde{x}=\tilde{y}+\tilde{s} \nu_{0}$, where $\tilde{s}, \hat{s} \in(-\infty, 0)$ and $\tilde{y}, \hat{y} \in P^{\lambda_{0}}\left(\nu_{0}\right)$. We shall show that

$$
|\tilde{s}-\hat{s}| \leq \cot \hat{\varepsilon}|\tilde{y}-\hat{y}| .
$$

This inequality shows that inside $H_{-}^{\lambda_{0}}\left(\nu_{0}\right)$, the outer boundary $\partial_{\text {out }}\left(\Gamma_{t}\right)$ is the graph of a function: $P^{\lambda_{0}} \rightarrow(-\infty, 0)$ with local Lipschitz constant $\cot \hat{\varepsilon}$.

Suppose inequality (2.1) is false; without loss of generality we may assume $\tilde{s}<\hat{s}<0$. Write the unit vector

$$
\nu_{k}:=\frac{\hat{x}_{k}-\tilde{x}}{\left|\hat{x}_{k}-\tilde{x}\right|} \rightarrow \nu:=\frac{\hat{x}-\tilde{x}}{|\hat{x}-\tilde{x}|} .
$$

We may compute

$$
\left\langle\nu, \nu_{0}\right\rangle=\frac{\left\langle\hat{y}-\tilde{y}, \nu_{0}\right\rangle+(\hat{s}-\tilde{s})}{\sqrt{|\hat{y}-\tilde{y}|^{2}+(\hat{s}-\tilde{s})^{2}}}>\frac{1}{\sqrt{\tan ^{2} \hat{\varepsilon}+1}}=\cos \hat{\varepsilon},
$$

which implies $\left\langle\nu_{k}, \nu_{0}\right\rangle>\cos \hat{\varepsilon}$, and hence $\operatorname{dist}\left(\nu_{k}, \nu_{0}\right)<\hat{\varepsilon}$, for $k$ sufficiently large. Note that $\left\langle\tilde{x}, \nu_{k}\right\rangle\left\langle\left\langle\hat{x}_{k}, \nu_{k}\right\rangle\right.$. Proposition 10 shows that $u_{t}$ is nonincreasing in $H_{-}^{\lambda_{0}}\left(\nu_{k}\right)$, which implies $0=u_{t}(\tilde{x}) \geq u_{t}\left(\hat{x}_{k}\right)>0$, a contradiction. This proves inequality (2.1) for any $\tilde{x}, \hat{x} \in \partial_{\text {out }}\left(\Gamma_{t}\right)$ in the open half-space $H_{-}^{\lambda_{0}}\left(\nu_{0}\right)$.

A similar argument shows that, inside $H_{-}^{\lambda_{0}}\left(\nu_{0}\right)$, the inner boundary $\partial_{i n}\left(\Gamma_{t}\right):=\Gamma_{t} \cap \overline{\Omega_{t}}$ is the graph of a second function: $P^{\lambda_{0}}\left(\nu_{0}\right) \rightarrow(-\infty, 0)$ satisfying the same local Lipschitz condition.

Applying Proposition 10 one more time, one sees that inside $H_{-}^{\lambda_{0}}\left(\nu_{0}\right)$, $\partial_{i n}\left(\Gamma_{t}\right)$ lies above $\partial_{\text {out }}\left(\Gamma_{t}\right)$ in terms of the $\nu_{0}$ direction. Since the Lipschitz bound $\cot \hat{\varepsilon}$ depends only on $\tilde{x}, \hat{x}$ and $\Gamma_{0}$, it is independent of $t$ and $F$.

Proof of Theorem 4. Note first that necessarily $\Lambda \geq 0$, since $\lambda_{\max }(\nu)+$ $\lambda_{\max }(-\nu) \geq 0$. 
Let $x_{0}$ be a point of $\Gamma_{t},\left|x_{0}\right|=: r_{0}>\Lambda$. Then for all $\nu \in S^{n}$, since $\lambda_{\max }(\nu) \geq-\Lambda$, we have $x_{0} \in H_{-}^{\max }(\nu)$ for all $\nu$ such that $\left\langle\nu, x_{0}\right\rangle<-\Lambda$. Write $x_{0}=r_{0} \theta_{0}$, where $\theta_{0} \in S^{n}$, and choose a unit tangent vector $e$ to $S^{n}$ at $\theta_{0}$. The inward radial unit vector $\nu_{0}=-\partial / \partial r$ satisfies $\left\langle\nu_{0}, x_{0}\right\rangle=-r_{0}<-\Lambda$, implying via Theorem 2, Part (2) that $\Gamma_{t}$ is a Lipschitz graph $r=\psi_{t}(\theta)$ in the radial direction in some neighborhood of $x_{0}$. The vector $\tau:=-r e-D \psi_{t}(e) \nu_{0}$ is tangent to $\Gamma_{t}$; according to Theorem 2, Part (2), $\tau$ is transverse to $\nu$ for all $\nu \in S^{n}$ satisfying $\left\langle\nu, x_{0}\right\rangle<-\Lambda$. Therefore

$$
-\frac{r D \psi_{t}(e)}{\sqrt{r^{2}+\left(D \psi_{t}(e)\right)^{2}}}=\left\langle\frac{\tau}{|\tau|}, x_{0}\right\rangle \geq-\Lambda
$$

Equivalently, after a little algebra

$$
D \psi_{t}(e) \leq \frac{r \Lambda}{\sqrt{r^{2}-\Lambda^{2}}}
$$

Proof of Corollary 7. According to Theorem 4, applied now in the level-set context ( $c f$. Remark 1), near any point outside of $\overline{B_{\Lambda}}(0), \partial_{0}\left(\Gamma_{t}\right)$ is locally a radial graph $r=\psi_{t}(\theta)$ where the Lipschitz function $\psi_{t}: S^{n} \rightarrow \mathbb{R}$ satisfies almost everywhere

$$
\left|D \psi_{t}\right| \leq \frac{r \Lambda}{\sqrt{r^{2}-\Lambda^{2}}}
$$

We define the constant $C_{0}$ by

$$
\pi=\int_{1}^{C_{0}} \frac{\sqrt{r^{2}-1}}{r} d r
$$

Write $x_{0}=r_{0} \theta_{0}$, where $r_{0}=\left|x_{0}\right|>C_{0} \Lambda$, and consider any other point $x_{1}=r_{1} \theta_{1} \in \partial_{0}\left(\Gamma_{t}\right)$. Write $\theta_{\perp}$ for a unit vector orthogonal to $\theta_{0}$ such that $\theta_{1}=\theta_{0} \cos s_{1}+\theta_{\perp} \sin s_{1}$ for some $0<s_{1} \leq \pi$. Consider the unit speed great-circle arc on $S^{n}$ from $\theta_{0}$ to $\theta_{1}$ :

$$
s \mapsto \theta(s):=\theta_{0} \cos s+\theta_{\perp} \sin s, 0 \leq s \leq s_{1} .
$$

Since, outside $\overline{B_{\Lambda}}(0), \partial_{0}\left(\Gamma_{t}\right)$ is a locally Lipschitz graph in the radial direction, there is a unique continuous lift from $\theta(s)$ to $r(s) \theta(s) \in \partial_{0}\left(\Gamma_{t}\right)$ on $0 \leq s<s^{*}$, where $s^{*}$ is the first value in $\left[0, s_{1}\right]$, if any, where $r\left(s^{*}\right)=\Lambda$, and $s^{*}=s_{1}$ otherwise. 
According to Theorem 4, we have

$$
\frac{d r}{d s} \geq-\frac{r(s) \Lambda}{\sqrt{(r(s))^{2}-\Lambda^{2}}}
$$

on $\left[0, s^{*}\right]$, with the initial condition $r(0)=r_{0}$. That is, $r(s)$ is a supersolution to an ordinary differential equation (evolute equation) whose exact solution $\tilde{r}(s)$ is given by $\tilde{r}(s)=\Lambda \sqrt{(\sigma(s))^{2}+1}$, where

$$
s=: \arctan \sigma(s)-\sigma(s)-\arctan \sigma_{0}+\sigma_{0}
$$

and $\sigma_{0}:=\sqrt{r_{0}^{2} \Lambda^{-2}-1}$. The hypothesis $r_{0}>C_{0} \Lambda$ implies $\sigma_{0}-\arctan \sigma_{0}>\pi$. On the other hand, $s \leq s^{*} \leq s_{1} \leq \pi$. This forces $\sigma(s) \geq 0$ and $r(s) \geq \Lambda$ on $\left[0, s^{*}\right]$. Hence $s^{*}=s_{1}$ and $r\left(s_{1}\right) \geq \Lambda$.

By a similar argument, we may show that

$$
\frac{r_{1}-r_{0}}{\Lambda} \geq C_{0}-1 \text {. }
$$

In fact, comparing any two intervals of the form $\left[r_{0}, r_{1}\right] \subset[0, \infty)$ on which $\arctan \sigma_{1}-\sigma_{1}-\arctan \sigma_{0}+\sigma_{0}=\pi$, where $r_{k}^{2}=: \Lambda^{2}\left(\sigma_{k}^{2}+1\right), k=0,1$, we see that the shortest such interval starts at $r_{0}=0$. This shows that $\partial_{0}\left(\Gamma_{t}\right)$ is a radial graph $r=\psi_{t}(\theta)$, where $\psi_{t}$ maps $S^{n}$ to an interval of length at most $\Lambda\left(C_{0}-1\right)$.

Now consider any point $x_{2}=r_{2} \theta_{2} \in \partial_{\text {out }}\left(\Gamma_{t}\right)$ (or in $\partial_{\text {in }}\left(\Gamma_{t}\right)$, respectively) with $r_{2}=\left|x_{2}\right|>\Lambda$. We may apply Theorem 6, part (2), with $\nu=-\theta_{2}$ and $\lambda_{0}=\Lambda \geq-\lambda_{\max }(\nu)$. Both $x_{2}=r_{2} \theta_{2}$ and $x_{3}:=\psi_{t}\left(\theta_{2}\right) \theta_{2}$ lie in $H_{-}^{\max }\left(-\theta_{2}\right)$, and therefore lie on the graph in the $\theta_{2}$-direction of the same function; hence $x_{2}=x_{3} \in \partial_{0}\left(\Gamma_{t}\right)$. This shows that outside $\overline{B_{\Lambda}(0)}, \partial_{\text {out }}\left(\Gamma_{t}\right)$ (or $\partial_{\text {in }}\left(\Gamma_{t}\right)$, respectively) agrees with $\partial_{0}\left(\Gamma_{t}\right)$.

\section{Application: Flow by $1 / H^{+}$.}

Several papers have been published recently which investigate properties of a hypersurface which expands with velocity equal to the reciprocal of mean curvature [G], [U1], [U2], [HI]. As a simple application of the results of the previous sections, we shall construct an "extended" solution $\Gamma_{t}$ for $1 / H^{+}$flow, and will show that as $t \rightarrow \infty$, and after suitable normalization, $\Gamma_{t}$ converges in Lipschitz norm to a round sphere or around annulus. Our approach is in a certain sense opposite to that of [G], [U2] and [HI], since we first show asymptotic roundness in the Lipschitz sense and only later, 
if at all, are in a position to prove that the curvatures are asymptotically constant. As in the recent result of Huisken and Ilmanen on the Penrose conjecture [HI], we do not need to assume the initial hypersurface is starshaped. In contrast to the solutions constructed in [HI], which result from a globally defined variational problem, our hypersurfaces are limits of solutions to local problems (see Remark 5 below.)

Consider an embedded, compact initial hypersurface $\Gamma_{0}$. In the next few paragraphs, we shall construct the solution $\Gamma_{t}$ to the extended evolution problem $V=1 / H^{+}$. $\Gamma_{t}$ will be the level set of the limit of viscosity solutions to a one-parameter family of geometric PDE's. We refer to this as an extended evolution problem, since the values of the right-hand side $1 / H^{+}$ are extended real numbers in $(0, \infty]$. Similarly, the corresponding PDE, the level sets of whose solutions are extended solutions to the $1 / H^{+}$-flow, may be called an extended PDE :

$$
\frac{\partial u}{\partial t}=\left\{\begin{array}{ll}
-\frac{|D u|}{\left(\operatorname{div} \frac{D u}{\mid D u}\right)^{+}} & \text {if } D u \neq 0 \\
0 & \text { if } D u=0
\end{array} .\right.
$$

As mentioned above, the solution $u$ will not necessarily be a viscosity solution, but instead will be the monotone limit of viscosity solutions of a family of regularized PDEs.

The first step is to regularize the problem. Given $\varepsilon>0$, we approximate $1 / H^{+}$with a smooth, strictly decreasing function $\Phi^{\varepsilon}: \mathbb{R} \rightarrow(0, \infty)$ such that $\Phi^{\varepsilon}(H)=1 / H$ if $H \geq \varepsilon$ and $1 / \varepsilon<\Phi^{\varepsilon}(H)<1+1 / \varepsilon$ for $H \in(-\infty, \varepsilon)$. In addition, we require that $\Phi^{\varepsilon}(H)$ be a nonincreasing function of $\varepsilon$.

Choose $K>0$, and define initial values as in Section 1 above:

$$
u_{0}(x)=\left\{\begin{array}{ll}
\operatorname{dist}\left(x, \Gamma_{0}\right) \wedge K & \text { if } x \in E_{0} \\
-\operatorname{dist}\left(x, \Gamma_{0}\right) & \text { if } x \in \Omega_{0}
\end{array},\right.
$$

where $\Omega_{0}$ is the bounded open set with $\partial \Omega_{0}=\Gamma_{0}$ and $E_{0}$ is their open complement. For each $\varepsilon>0$, there is a unique, continuous viscosity solution $u^{\varepsilon}$ to the equation

$$
\frac{\partial u^{\varepsilon}}{\partial t}=-\left|D u^{\varepsilon}\right| \Phi^{\varepsilon}\left(\operatorname{div} \frac{D u^{\varepsilon}}{\left|D u^{\varepsilon}\right|}\right)
$$

for $(x, t) \in \mathbb{R}^{n+1} \times(0, \infty)$ satisfying the initial condition $u^{\varepsilon}(x, 0)=u_{0}(x)$ [CGG]. Observe that, since $\Phi^{\varepsilon}(H)$ is a nonincreasing function of $\varepsilon, u^{\varepsilon}(x)$ is a 
nondecreasing function of $\varepsilon$. Namely, if $0<\varepsilon_{0}<\varepsilon$, then $u^{\varepsilon_{0}}$ is a subsolution of the equation (3.3). Also, $u^{\varepsilon}$ is bounded above by the constant $K$ and below by the infimum of $u_{0}$ on $\Omega_{0}$. Therefore, $u^{\varepsilon}$ converges pointwise and monotonically to a function $u$ as $\varepsilon \rightarrow 0$. We define the zero level set $\Gamma_{t}$ of $u(\cdot, t)$ to be an extended solution of $1 / H^{+}$flow.

The solution $u$ will in general have discontinuities, and we will not claim it is a viscosity solution of the extended PDE (3.1). However, it will have the approximate-symmetry properties we have shown in Section 2 above for viscosity solutions of geometric PDE's. To see this, suppose $\left(-\infty, \lambda_{0}\right)$ is admissible for the initial hypersurface $\Gamma_{0}$ with respect to a unit vector $\nu \in S^{n}$. Then we may apply Proposition 10 to the viscosity solutions $u^{\varepsilon}$ of the regularized PDE (3.3). The conclusion of Proposition 10 is that $u^{\varepsilon}$ is nonincreasing in the $\nu$ direction on $H_{-}^{\lambda_{0}}(\nu)$. Since $u^{\varepsilon} \rightarrow u$ pointwise, it follows that $u$ itself is nonincreasing in the $\nu$ direction on $H_{-}^{\lambda_{0}}(\nu)$. We conclude that the level set $\Gamma_{t}$ of $u(\cdot, t)$ satisfies the conclusions of Theorem 6 , and all the hypotheses of Corollary 7 except the existence of $x_{0} \in \Gamma_{t}$ with $\left|x_{0}\right|>C_{0} \Lambda$.

We shall show next that the zero level set $\Gamma_{t}$ of $u_{t}$ expands outward toward infinity as $t \rightarrow \infty$. Without loss of generality, suppose that the origin $O$ of $\mathbb{R}^{n+1}$ lies inside $\Omega_{0}$, and choose a radius $\rho_{0}$ such that $B_{\rho_{0}}(O) \subset \Omega_{0}$. Define $v_{0}: \mathbb{R}^{n+1} \rightarrow \mathbb{R}$ to be the signed distance function from $\partial B_{\rho_{0}}(O)$ :

$$
v_{0}(x):=\left(|x|-\rho_{0}\right) \wedge K .
$$

Note that $v_{0}(x) \geq u_{0}(x)$. Starting from the initial condition $v^{\varepsilon}(x, 0)=v_{0}(x)$, there is a unique solution $v^{\varepsilon}(x, t)$ to the regularized PDE (3.3) for $(x, t) \in$ $\mathbb{R}^{n+1} \times[0, \infty)$. Since $v^{\varepsilon}$ and $u^{\varepsilon}$ are viscosity solutions of the same PDE (3.3), we have $v^{\varepsilon}(x, t) \geq u^{\varepsilon}(x, t)$ for all $(x, t) \in \mathbb{R}^{n+1} \times[0, \infty)$ (see e.g. Theorem 4.1 of [CGG]). It follows that $u(x, t) \leq u^{\varepsilon}(x, t) \leq v^{\varepsilon}(x, t)$ for all $t \geq 0$. But the zero level set of $v^{\varepsilon}(\cdot, t)$ is $\partial B_{\rho(t)}(O)$, where $\rho(t)=\rho(t, \varepsilon)$ satisfies the ODE

$$
\frac{d \rho}{d t}=\Phi^{\varepsilon}\left(\frac{n}{\rho(t)}\right)=\frac{\rho(t)}{n} \text { for } \rho(t) \leq \frac{n}{\varepsilon},
$$

with initial condition $\rho(0, \varepsilon)=\rho_{0}$. We have $v^{\varepsilon}(x, t)<0$ whenever $|x|<$ $\rho(t, \varepsilon)$. For $\varepsilon \leq\left(n / \rho_{0}\right) \exp (-t / n)$, we find $\rho(t, \varepsilon)=\rho_{0} \exp (t / n)$ independent of $\varepsilon$, by means of a straightforward computation. This implies that $u_{t}=$ $u(\cdot, t)<0$ on a ball $B_{\rho(t)}(O)$ of exponentially growing radius. Therefore $\Gamma_{t}$ lies entirely outside an arbitrarily large ball for large $t$, and the remaining hypothesis of Corollary 7 is satisfied for sufficiently large $t$. We conclude that 
$\partial_{\text {out }}\left(\Gamma_{t}\right)$, after rescaling to enclose volume equal to $\operatorname{Vol}\left(B_{1}(O)\right)$, converges at an exponential rate to a round sphere in the Lipschitz norm. The same conclusion holds for $\partial_{i n}\left(\Gamma_{t}\right)$.

Remark 3. In order to obtain convergence of the curvatures of $\partial_{i n}\left(\Gamma_{t}\right)$ and of $\partial_{\text {out }}\left(\Gamma_{t}\right)$ to constants, it would be necessary to derive curvature estimates for level sets of $u^{\varepsilon}$, for example. Such estimates are a topic for another paper.

Remark 4. We would like to show that these extended solutions satisfy a weak barrier principle. Suppose that $\Gamma_{0}$ and $\widetilde{\Gamma}_{0}$ are compact hypersurfaces in $\mathbb{R}^{n+1}$ and let $\Omega_{0}$ resp. $\widetilde{\Omega}_{0}$ be a bounded union of components of the complement. Choose $u_{0}: \mathbb{R}^{n+1} \rightarrow \mathbb{R}$ and $\widetilde{u}_{0}: \mathbb{R}^{n+1} \rightarrow \mathbb{R}$ to be the signed distance functions from $\Gamma_{0}$ resp. $\widetilde{\Gamma}_{0}$. Let $u_{t}$ resp. $\widetilde{u}_{t}$ be the solutions of the extended PDE (3.1) and let $\Gamma_{t}, \widetilde{\Gamma}_{t}$ be their zero sets at time $t$. If $\widetilde{\Gamma}_{0}$ lies inside $\Omega_{0}$, or more generally if $\widetilde{\Omega}_{0} \subset \Omega_{0}$, then for all $t>0, \widetilde{\Omega}_{t} \subset \Omega_{t}$. That is, one extended solution $\Gamma_{t}$ of $1 / H^{+}$flow acts as a barrier to any other extended solution $\widetilde{\Gamma}_{t}$.

The proof is similar to the proof above of approximate-symmetry properties. As in the proof of Lemma 9, the inclusion $\widetilde{\Omega}_{0} \subset \Omega_{0}$ implies that $\tilde{u}_{0} \geq u_{0}$. The weak maximum principle then allows us to conclude that for all $t>0, \widetilde{u}_{t} \geq u_{t}$, which implies that $\widetilde{\Omega}_{t} \subset \Omega_{t}$, as required.

The reader might well ask about the consistency of our definition of extended solutions. The following result is clear.

Proposition 11. If $M^{n}$ is a compact n-manifold and $X: M^{n} \times[0, T) \rightarrow$ $\mathbb{R}^{n+1}$ is a classical, embedded $C^{2}$ solution to the inverse mean curvature flow

$$
\frac{\partial X}{\partial t}=\frac{1}{H} N
$$

with $H>0$ ( $N$ is the unit outward normal), then our extended solution $u$ with the same initial data $\left\{x: u_{0}(x)=0\right\}=X(0)\left(M^{n}\right)$ is equal to the classical solution:

$$
\left\{x: u_{t}(x)=0\right\}=X(t)\left(M^{n}\right)
$$

for all $t \in[0, T)$.

Proof. Given any $\tau<T$, let $\varepsilon_{\tau}=\min _{M^{n} \times[0, \tau]} H$. Then equation (3.3) defines a viscosity solution $u=u^{\varepsilon}$ (independent of $\varepsilon$ ) to (5) on $\mathbb{R}^{n+1} \times[0, \tau]$ with 
$u(0)=u_{0}$ for any $\varepsilon \in\left(0, \varepsilon_{\tau}\right]$ and the proposition follows from the uniqueness of the solution (see [CGG]).

Remark 5. By means of a fairly simple example, we would like to compare our notion of "extended" solutions to $1 / \mathrm{H}^{+}$-flow with the variational solutions recently introduced by Huisken and Ilmanen [HI]. Let $\Gamma_{0}$ be the union of two disjoint spheres lying outside each other. Then for $t$ in a certain time interval $[0, T]$, an embedded solution $\Gamma_{t}$ of $1 / H$ flow will continue to exist. In fact, $\Gamma_{t}$ will be the union of two spheres corresponding to the two components of $\Gamma_{0}$. If one of the original components of $\Gamma_{0}$ has radius $r_{0}$, then the corresponding sphere in $\Gamma_{t}$ has the same center and radius $r_{0} e^{t}$. This solution ceases to be embedded at the first time $T$ when the two spheres of $\Gamma_{t}$ intersect. According to Proposition 11, $\Gamma_{t}$ will be the extended solution of $1 / H^{+}$-flow for all $0 \leq t<T$. In contrast, Example 1.5 of $[\mathrm{HI}]$ indicates how the Huisken-Ilmanen solution of $1 / H^{+}$-flow agrees with $\Gamma_{t}$ only for $t<T^{\prime}$, where $T^{\prime}$ is strictly less than $T$. Specifically, if the two spheres which comprise $\Gamma_{t}$ are sufficiently close together, then there will be a unique catenoid tangent to both of them, at small circles $C_{1}$ and $C_{2}$, one circle in each sphere of $\Gamma_{t}$. Then $T^{\prime}$ is the time at which the area of the segment of the catenoid between $C_{1}$ and $C_{2}$ equals the area of the union of the two spherical caps of $\Gamma_{t}$ having the $C_{1}$ and $C_{2}$ as boundary.

\section{Examples.}

\subsection{Two circles.}

Bellettini and Paolini have proposed a simple and intriguing initial condition consisting of $\Gamma_{0}=$ two circles in $\mathbb{R}^{2}$ bounding disjoint disks. They allowed $\Gamma_{0}$ to flow as a level set, that is, $\Gamma_{t}=\{x: u(x, t)=0\}$, where $u$ satisfies equation (1.1) with $F$ in the form $F(\kappa, t)=\kappa+g(t)$, where $\kappa$ is the curvature of $\Gamma_{t}$. For certain choices of the forcing term $g(t)$, the initial radii $r_{10}$ and $r_{20}$, and the distance between the centers of the two circles of $\Gamma_{0}$, they show that $\Gamma_{t}$ has positive Lebesgue measure in $\mathbb{R}^{2}$ after a time $t^{*}>0$ $[\mathrm{BP}]^{1}$. After rescaling, we may assume that the two circles comprising $\Gamma_{0}$ are $\partial B_{r_{10}}((-1,0))$ and $\partial B_{r_{20}}((+1,0))$. Let $r_{1}(t)$ and $r_{2}(t)$ be the solutions of the ODE

$$
r_{i}^{\prime}(t)=-\left(r_{i}(t)\right)^{-1}+g(t), \quad 0 \leq t<T
$$

\footnotetext{
"That is, "ballooning" or "fattening" of the solution occurs at time $t^{*}$.
} 
with initial conditions

$$
r_{i}(0)=r_{i 0} \quad(i=1,2),
$$

and where $T \in(0, \infty]$ is the smaller of the blow up times for the two ODEinitial value problems. Belletini and Paolini show that ballooning occurs at time $t^{*}<T$ provided that $g(t) \geq 0$ for all $t$ and that the sum of the solutions $r_{1}(t)+r_{2}(t)$ reaches the maximum value 2 at $t=t^{*}$.

We shall apply Theorem 6 to show that for each $t>0, \Gamma_{t}$ is the closed set between two locally Lipschitz graphs:

$$
\Gamma_{t}=\left\{\left(x_{1}, x_{2}\right): \varphi_{\text {in }}^{t}\left(x_{1}\right) \leq\left|x_{2}\right| \leq \varphi_{\text {out }}^{t}\left(x_{1}\right)\right\}
$$

where $\varphi_{\text {in }}^{t}, \varphi_{\text {out }}^{t}: \mathbb{R} \rightarrow \mathbb{R}$ are continuous and uniformly Lipschitz away from the $x_{1}$-axis. The Lipschitz bounds are independent of $t$, and in fact take the simple form

$$
-1 \leq \varphi^{t}\left(x_{1}\right) \frac{d}{d x_{1}} \varphi^{t}\left(x_{1}\right)+x_{1} \leq 1,
$$

for both $\varphi_{\text {in }}^{t}$ and $\varphi_{\text {out }}^{t}$. The local Lipschitz estimate (4.1) holds whether or not ballooning occurs; if $\Gamma_{t}$ is a smooth curve, then $\varphi_{\text {in }}^{t} \equiv \varphi_{\text {out }}^{t}$.

Note in particular that inequality (4.1) forces $\Gamma_{t}$, or the inner or outer boundary of a ballooned $\Gamma_{t}$, to have a vertical tangent line whenever it crosses the $x_{1}$-axis outside the closed interval $-1 \leq x_{1} \leq 1$, but that the Lipschitz bounds lose all force near $(-1,1)$ on the $x_{1}$-axis.

To prove inequality (4.1), we first compute $\lambda_{\max }(\nu)$ for an arbitrary $\nu=(\cos \theta, \sin \theta) \in S^{1}$. Given $\lambda \in \mathbb{R}$, we observe that the portion of a given circle in $\Gamma_{0} \cap H_{-}^{\lambda}(\nu)$ reflects to the interior of the circle if and only if the center of the circle lies in $H_{+}^{\lambda}(\nu)$. That is, $\lambda$ is admissible for $\Gamma_{0}$ with respect to $\nu=(\cos \theta, \sin \theta)$ if and only if both centers $( \pm 1,0)$ lie in $\overline{H_{+}^{\lambda}}$, that is, $\lambda \leq-|\cos \theta|$. Thus $\lambda_{\max }(\nu)=-|\cos \theta|$. According to Theorem 6, for any $t \in(0, T), \lambda$ will remain admissible for $\Gamma_{t}$ with respect to $\nu$ whenever $\lambda \leq-|\cos \theta|$.

Now suppose $\nu_{0}=\left(\cos \theta_{0}, \sin \theta_{0}\right)$, and consider a point $x=\left(x_{1}, x_{2}\right) \in$ $H_{-}^{\max }\left(\nu_{0}\right)$, which is equivalent to

$$
\left\langle x, \nu_{0}\right\rangle<-\left|\cos \theta_{0}\right| .
$$

Then inequality (4.2) remains true for all $\nu \in S^{1}$ sufficiently close to $\nu_{0}$, that is,

$$
\langle x, \nu\rangle<-|\cos \theta|=\lambda_{\max }(\nu)
$$


for all $\theta$ near $\theta_{0}$, writing $\nu=(\cos \theta, \sin \theta)$. Thus, according to Part 2 of Theorem 6, for each $\nu$ satisfying (4.3) and for all $t \geq 0, \Gamma_{t} \cap H_{-}^{\max }(\nu)$ is the closed set between two locally Lipschitz graphs in the direction $\nu$.

In particular, with $\theta_{0}=-\pi / 2$, we have $\lambda_{\max }\left(\nu_{0}\right)=0$, and inequality (4.2) holds whenever $x_{2}>0$. That is, $H_{-}^{\max }(0,-1)$ is the open upper half-plane. This allows us to conclude, for any given $t \in(0, T)$, that the intersection of $\Gamma_{t}$ with the upper half-plane is the relatively closed set

$$
\left\{x: x_{2}>0, \varphi_{\text {in }}^{t}\left(x_{1}\right) \leq x_{2} \leq \varphi_{\text {out }}^{t}\left(x_{1}\right)\right\}
$$

for some functions $\varphi_{\text {in }}^{t}, \varphi_{\text {out }}^{t}: \mathbb{R} \rightarrow[0, \infty)$ which are locally Lipschitz away from zeroes.

We next show the sharp Lipschitz bound (4.1). With either $\varphi=\varphi_{\text {in }}^{t}$ or $\varphi=\varphi_{\text {out }}^{t}$, consider any point $x=\left(x_{1}, x_{2}\right)$ in the upper half-plane on the inner or outer boundary, respectively, of $\Gamma_{t}: x_{2}=\varphi\left(x_{1}\right)>0$. For small $\varepsilon>0$, write $x^{+}=\left(x_{1}, x_{2}+\varepsilon\right)$ and $x^{-}=\left(x_{1}, x_{2}-\varepsilon\right)$. By Theorem 6 , for any $\nu=(\cos \theta, \sin \theta)$ satisfying inequality (4.3), $x \in H_{-}^{\max }(\nu)$ and $\Gamma_{t} \cap H_{-}^{\max }(\nu)$ is the relatively closed set between two Lipschitz graphs in the $\nu$-direction (as well as in the $\nu_{0}$-direction). We restrict $\theta$ to the interval $(-\pi, 0)$ and note that inequality (4.3) holds for $\theta$ in an interval on both sides of $\theta_{0}=-\pi / 2$. Choose $\varepsilon$ small enough that $x^{ \pm} \in H_{-}^{\max }(\nu)$. Then the sign (0 or +1 , resp.) of $u_{t}$ is constant along the (upward) ray $x^{+}-s \nu, 0 \leq s<\infty$. Similarly, the sign ( -1 or 0 , resp.) of $u_{t}$ is constant along the segment $x^{-}+s \nu, 0 \leq s<-\langle x, \nu\rangle-|\cos \theta|+\varepsilon \sin \theta$. In terms of the function $\varphi$, this means that a line segment starting from $x^{+}=\left(x_{1}, \varphi\left(x_{1}\right)+\varepsilon\right)$ and having slope $\tan \theta$ lies above the graph of $\varphi$, and that a line segment starting from $x^{-}$with the same slope lies below the graph of $\varphi$. Note, using inequality (4.3), that the lengths of these segments may be chosen independent of $\varepsilon$. Letting $\varepsilon \rightarrow 0$ gives bounds for $\varphi^{\prime}\left(x_{1}\right)$, assuming for convenience that this derivative exists (otherwise, we find the same bounds on difference quotients with sufficiently small denominators). The bound states that $\varphi^{\prime}\left(x_{1}\right) \leq \tan \theta$ when $\tan \theta \geq 0$, i.e., when $\theta \leq-\pi / 2$. In this case, inequality (4.3) holds whenever $x_{2} \tan \theta>1-x_{1}$, which shows $\varphi\left(x_{1}\right) \varphi^{\prime}\left(x_{1}\right) \leq 1-x_{1}$, which is the right-hand side of inequality (4.1). When $\tan \theta \leq 0$, i.e. $-\pi / 2 \leq \theta$, this is a lower bound $\varphi^{\prime}\left(x_{1}\right) \geq \tan \theta$, for all $\theta$ such that $x_{2} \tan \theta<1-x_{1}$, and the other side of inequality (4.1) follows. This proves the Lipschitz bound (4.1). Moreover, the estimate (4.1) is sharp. In fact, equality holds on the right-hand side of (4.1) for a circle centered at $(1,0)$, and on the left-hand side for a circle centered at $(-1,0)$. In particular, this is the case for $\Gamma_{t}$, $0 \leq t<t^{*}$. 
We next consider reflections $\sigma^{\lambda}\left(\nu_{0}\right)$ with $\nu_{0}=(0,1)$; as we have seen, $H_{-}^{\max }(0,1)$ is the lower half-plane. We shall show that $\Gamma_{t}$ is symmetric under reflection $\sigma^{\max }\left(\nu_{0}\right)$ in the $x_{1}$-axis. From the proof of Part 1 of Theorem $6,(-\infty, 0]$ is admissible for $u_{t}$ with respect to $\nu_{0}=(0,1)$; recall $\Gamma_{t}=\left\{x: u_{t}(x)=0\right\}$. In particular, $u_{t}$ is a nonincreasing function of $x_{2} \in(-\infty, 0]$ for each fixed $x_{1} \in \mathbb{R}$. Further, since $\lambda=0$ is admissible for $u_{t}$ with respect to $\nu_{0}=(0,1)$, we have $u_{t}\left(x_{1}, x_{2}\right) \leq u_{t}\left(x_{1},-x_{2}\right)$ for all $x_{2} \geq 0$, by Definition 6. On the other hand, $\lambda=0$ is also admissible for $u_{t}$ with respect to $-\nu_{0}=(0,-1)$, so that $u_{t}\left(x_{1}, x_{2}\right) \geq u_{t}\left(x_{1},-x_{2}\right)$ for all $x_{2} \geq 0$. Therefore $u_{t}\left(x_{1}, \cdot\right)$ is an even function for all $x_{1} \in \mathbb{R}$, and $\Gamma_{t}$ is invariant under reflection in the $x_{1}$-axis.

Higher Dimensions. Results analogous to $[\mathrm{BP}]$ have been recently published for hypersurfaces in $\mathbb{R}^{n+1}$. Koo $[\mathrm{K}]$ has shown that if an immersed surface $\Sigma_{t}$ evolves by $V=-H+g(t)$ for some nonnegative function $g(t)$, and touches itself from the outside at time $t^{*}$ without crossing, then the corresponding generalized solution $\Gamma_{t}$ has positive Lebesgue measure for times $t$ in an interval $\left(t^{*}, t^{*}+\delta\right)$.

In particular, fattening occurs for the initially smooth example of a pair of evolving spheres whose centers lie at an appropriately chosen distance. An application of Part 1 of Theorem 6, completely analogous to the case $n=1$ just discussed, shows that for all $t>t^{*}, \Gamma_{t}$ is the closed set between the hypersurfaces of revolution generated by two graphs $r=\varphi_{\text {in }}^{t}\left(x_{1}\right)$ and $r=\varphi_{\text {out }}^{t}\left(x_{1}\right)$, in cylindrical coordinates about the axis containing the centers of the two initial spheres. The functions $\varphi_{\text {in }}^{t}$ and $\varphi_{\text {out }}^{t}: \mathbb{R} \rightarrow[0, \infty)$ satisfy the estimate (4.1) and hence are locally Lipschitz away from zeroes.

\subsection{Non-Lipschitz Example.}

The purpose of this example is to highlight the distinction between the first and second parts of Theorems 2 and 6 . Namely, if a point $x$ is in $H_{-}^{\lambda}\left(\nu_{0}\right)$ but $\langle x, \nu\rangle>\lambda_{\max }(\nu)$ for all $\nu$ in a punctured neighborhood of $\nu_{0}$, then Part 1 of the theorem applies near $x$, but not Part 2. In this example, the conclusion of Part 2 fails: for a certain $t_{0}>0, \Gamma_{t}$ is not a Lipschitz submanifold at $x$.

We begin with the case $n=1$ of an evolving curve in the plane. Let $\left(x_{0}, x_{1}\right)$ be Euclidean coordinates for $\mathbb{R}^{2}$. Choose four line segments parallel to the $x_{0}$-axis, all ending at the line $x_{0}=-1$, the outer two starting at $x_{0}=+1$, and the inner two at the $x_{1}$-axis. Now add four semicircles to complete a connected, embedded $C^{1}$ curve $\Gamma_{0}$. Write $\nu_{0}=(1,0)$. Then for $\nu \neq \pm \nu_{0}, \lambda=\lambda_{\max }(\nu)$ is the largest value such that the centers of all four 
semicircles lie in $\bar{H}_{+}^{\lambda}(\nu)$.

In particular, as $\nu \rightarrow \nu_{0}, \lambda_{\max }(\nu) \rightarrow-1$. However, $\lambda_{\max }\left(\nu_{0}\right)=0$. Thus, even though $(-\infty, 0)$ is admissible for $\nu_{0}$, it cannot be concluded from Theorem 6, Part (2) that $\Gamma_{t}$, or its inner and outer boundaries, are Lipschitz hypersurfaces inside the half-plane $H_{-}^{0}\left(\nu_{0}\right)=H_{-}^{\max }\left(\nu_{0}\right)$.

In fact, this conclusion fails, at least for the fully degenerate case of the Hamilton-Jacobi equations. If the curve moves outward with prescribed constant velocity $-F\left(\kappa_{1}, \ldots, \kappa_{n}, t\right)=1$, then the inner two line segments will collide at a certain time $t^{*}>0$, where $2 t^{*}$ is the distance between the inner segments of $\Gamma_{0}$. The level set $\Gamma_{t^{*}}$ is singular, since it includes a triple point where two semicircles are externally tangent and are continued by a segment of their common tangent. Moreover, this line segment has the bounded open set $\Omega_{t^{*}}$ on both sides.

This example shows that Lipschitz or even topological regularity of $\Gamma_{t}$, or its inner and outer boundaries, inside the open half-space $H_{-}^{\max }(\nu)$ is not guaranteed. Nevertheless, regardless of the choice of $\Gamma_{0}$ and $F$, a limited regularity follows from Proposition 10: $\Gamma_{t} \cap H_{-}^{\max }(\nu)$ is the closed set between the graphs of two semicontinuous functions.

Higher Dimensions. If the original curve $\Gamma_{0}$ is chosen to be symmetric about the $x_{0}$-axis, then rotation about the $x_{0}$-axis forms a hypersurface of $\mathbb{R}^{n+1}$ such that the solution of the outward Hamilton-Jacobi flow $F=-1$ starting from this hypersurface has analogous properties to those described above for $n=1$. For example, after a certain time $t^{*}>0, \Gamma_{t^{*}}$ contains the segment $0 \leq x_{0}<1$ of the $x_{0}$-axis, which is also in the interior of $\overline{\Omega_{t^{*}}}$.

With the inward Hamilton-Jacobi flow $F=+1$, and with the same rotationally symmetric initial hypersurface $\Gamma_{0}$, the evolving level set becomes singular at some positive time $t^{* *}: \Gamma_{t^{* *}}$ includes a cylindrical segment which bounds $E_{t^{* *}}$ on both sides.

\section{References.}

[A1] A.D. Aleksandrov, Uniqueness theorems for the surfaces in the large, I, Vestnik Leningrad Univ. 11 (1956), 5-17; English transl. in Amer. Math. Soc. Transl. 21 (1962), 341-354.

[A2] A.D. Aleksandrov, Uniqueness theorems for the surfaces in the large, II, ibid. 12 (1957), 15-44; English transl. pp. 354-388.

[A3] A.D. Aleksandrov, Uniqueness theorems for the surfaces in the large, III, ibid. 13 (1958a), 14-26; English transl. pp. 389-403. 
[A4] A.D. Aleksandrov, Uniqueness theorems for the surfaces in the large, $V$, ibid. 13 (1958b), 5-8; English transl. pp. 412-416.

[AVo] A.D. Aleksandrov and Ju.A. Volkov, Uniqueness theorems for the surfaces in the large, IV, ibid. 13 (1958), 27-34; English transl. pp. 403-411.

[AVe] S. Angenent and J.J.L. Velázquez, in preparation.

[AIC] S. Angenent, T. Ilmanen, and D. Chopp, A Computed Example of Nonuniqueness of Mean Curvature Flow in $\mathbb{R}^{3}$, Comm. Partial Differential Eq. 20 (1995), 1937-1958.

[BP] G. Belletini and M. Paolini, Two Examples of Fattening for the Mean Curvature Flow with a Driving Force, Atti Accad. Naz. Lincei Cl. Sci. Fis. Mat. Natur. Rend. 9 (1994), Mat. Appl. 5, 229-236.

[CGG] Y.G. Chen, Y. Giga, and S. Goto, Uniqueness and Existence of Viscosity Solutions of Generalized Mean Curvature Flow Equations, J. Differential Geom. 33 (1991), 749-786.

[CB1] B. Chow and R. Gulliver, Aleksandrov reflection and nonlinear evolution equations, I: The $n$-sphere and $n$-ball, Calculus of Variations and Partial Differential Equations 4 (1996), 249-264.

[CB2] B. Chow and R. Gulliver, Aleksandrov reflection and nonlinear evolution equations, II: manifolds with reflectional symmetry, in preparation.

[ES] L.C. Evans and J. Spruck, Motions of Level Sets by Mean Curvature, I, J. Differential Geom. 33 (1991), 635-681.

[HI] G. Huisken and T. Ilmanen, The Inverse Mean-Curvature Flow and the Riemannian Penrose Inequality, to appear in J. Differential Geom.

[G] C. Gerhardt, Flow of Nonconvex Hypersurfaces into Spheres, J. Differential Geom. 32 (1990), 299-314.

[K] Y. Koo, A Fattening Principle for Fronts Propagating by Mean Curvature plus a Forcing Term, Comm. Partial Differential Eq. 24 (1999), 1035-1053.

[U1] J.I.E. Urbas, An expansion of convex hypersurfaces, J. Differential Geom. 33 (1991), 91-125; Correction to, ibid. 35 (1992), 763-765.

[U2] J.I.E. Urbas, On the Expansion of Starshaped Hypersurfaces by Symmetric Functions of their Principal Curvatures, Math. Zeit. 205 (1990), 355-372. 
ReCeived December 17, 1998. 